

\section{Cambios educativos en los procesos de lectura digital: la pedagogía del ciberespacio como estrategia de procesa- miento de contenidos en la era de internet}

Educational changes in the processes of digital reading: pedagogy of cyberspace as processing strategy contained in the Internet age DOI: $10.15213 /$ REDES.N12.P222

DIEGO APOLO, MARC BAYÉS Y ANDRÉS HERMANN

La red y los ordenadores no son buenos

o malos per se; todo depende de cada

práctica particular, en cada contexto.

Daniel Cassany

\section{RESUMEN}

La eclosión de las tecnologías digitales y la comunicación, a finales del siglo XX y primer decenio del siglo XXI, ha posibilitado que el uso de las herramientas digitales permitan re-pensar los modelos educativos vigentes; en este sentido, se tiene en cuenta la necesidad de emprender procesos de alfabetización digital que no sólo se circunscriban al manejo artefactual de la red internet y de los ordenadores, sino también al desarrollo del pensamiento crítico y creativo que se encuentra en esta nueva ecología de medios.

Las formas de lectura que se dan con el uso de las tecnologías analógicas se caracterizan por ser de tipo lineal o secuencial, mientras las formas de lectura que se da con las tecnologías digitales brindan la posibilidad de tener una lectura e interpretación de los datos de forma reticular o bifurcada, en donde el usuario puede elegir la ruta de la trama o historia.

Se propone, asimismo, la pedagogía del ciberespacio como teoría de enseñanza y aprendizaje que permite ubicar estrategias para el procesamiento de datos e información, conocimientos y aprendizajes de los lenguajes o narrativas virtuales que se encuentran de manera natural la red internet y entornos digitales.

PALABRAS CLAVE: NARRATIVAS DIGITALES, HIPERTEXTO, PEDAGOGÍA DEL CIBERESPACIO, ENTORNO DIGITAL, TECNOLOGÍAS EDUCATIVAS. 


\section{ABSTRACT}

The emergence of new information and communications technology in the decade of the 9os in the twentieth century and in the first decade of the 21st century, has enabled the use of communication tools reconfiguring the current educational model. In this sense, the need to undertake processes of digital literacy are not only confined to artifactual net management Internet and computers (hardware and software), but also the evolution of critical and creative thinking found in this new technological ecosystem.

Forms of reading that occur within the use of analog technologies are characterized by linear or sequential-type, while forms of reading that occur with digital technologies, provide the possibility of net shaped or branched data interpretation where the user can choose the path of the plot or story.

This raises questions in regards to a pedagogy of cyberspace and a theory of teaching and learning strategies that would put data processing and information, knowledge and learning of languages or virtual narratives which can open a natural field for web use and digital environments.

KEYWORDS: DIGITAL NARRATIVE, HYPERTEXT PEDAGOGY OF CYBERSPACE, DIGITAL ENVIRONMENT, EDUCATIONAL TECHNOLOGIES.

\section{INTRODUCCión}

Cuando la red internet apareció al público, esta red de redes se caracterizó por poseer un uso complejo, es decir, que solo los denominados programadores o "web masters" podían ser productores de contenidos. A este momento histórico se le conoce como la web 1.o. En el año 2004, Tim O’Reilly acuñaría el término web 2.0, como respuesta para brindar una denominación a los cambios que se venían dando con la red internet, desde la perspectiva de que la web poseía un uso más intuitivo, ya que sus nuevas herramientas, como blogs, wikis, entornos digitales y otras plataformas podían ser gestionadas por los usuarios. Es decir, se da un paso decisivo en las audiencias: de consumidores a productores activos de contenidos, ideas y significados en la red internet.

En el actual momento histórico es necesario hablar de la web 3.0, una categoría de análisis que todavía no está institucionalizada. La reflexión en torno a esta categoría es incipiente, lo que no quita valor al hecho de que se pueda definir como el momento en que se halla la red internet en la actualidad: una 
red intuitiva, por ser semántica, y enriquecida, desde la perspectiva de que son los usuarios-productores los que alimentan la información en los entornos digitales.

El análisis presentado con respecto a los cambios que ha tenido la red internet estas dos últimas décadas, con el paso de la web 1.0 a la web $2.0 \mathrm{y}$ la web 3.0, permite ubicarnos en la reflexión central del objeto de estudio de este trabajo, a saber: el proceso de transformación de la educación en la era digital y la reflexión sobre la lectura en la sociedad red.

En este contexto, se están tejiendo nuevos discursos, relatos y narrativas digitales que condicionan profundamente la lectura en los dispositivos digitales y que posibilitan una comunicación y educación más interactiva y estimulante.

En cuanto al sistema educativo se refiere, el advenimiento de la Posmodernidad y la sociedad en red no ha significado un cambio estructural en los sistemas educativos, sino que se han mantenido por el momento los enfoques neoliberales funcionalistas en la enseñanza, aunque con una nueva realidad insoslayable: la tecnología ha entrado de forma masiva en el aula. Esos cambios tecnológicos han demandado y requieren "especialistas y expertos" seleccionados y formados "en un sistema educativo en plena expansión" (Bonal, 1998:38).

Krawczyk (2002) menciona que las propuestas educativas nacidas desde (...) organismos internacionales [ONU, OCDE, CEPAL, etc.] han condicionado su funcionamiento y despliegue de recursos. Los sistemas educativos de estas instituciones han desplegado y masificado el uso y manejo de la técnica, mas no una conveniente reflexión que favorezca el establecimiento de caminos de construcción conjunta entre lo técnico y lo analítico, que vaya más allá de la predominancia de lo técnico en detrimento de lo reflexivo, lo pensado.

Rama (2004) señala a ese respecto que "el actual modelo pedagógico reproduce el conocimiento, pero no siempre ayuda a generar nuevos conocimientos, ni siquiera apropiarse de una mayor cantidad de los conocimientos existentes" (RAMA 2004:48). En otras palabras, la tecnología invadió las aulas con un carácter instrumental. "La imagen del conocimiento reflejaba que el compromiso y la visión de la educación eran una réplica de las tareas" (Bauman, 2007, p.26), es decir, las actividades de clase, cuyo método de aprendizaje se basaba en la repetición y memorización de los supuestos impartidos por los docentes.

El docente era la única autoridad del conocimiento y no contaba con la interacción y la construcción de conocimiento conjunto. Lo que para Jenkins (2008, p.188) se refleja en las escuelas es que "siguen confinadas a un modelo 
de aprendizaje autónomo que contrasta drásticamente con los tipos de aprendizaje que se precisan conforme ingresan los alumnos en las nuevas culturas del conocimiento". Es un modelo, en suma, con nuevos soportes digitales, pero profundamente anclado en lo tradicional.

En este contexto son muy necesarias las reflexiones sobre esta nueva realidad porque la transformación tecnológica en las instituciones educativas es un hecho evidente, un reflejo de una realidad social mucho más compleja. Steiner señala al respecto que:

La computación, la teoría y la búsqueda de la información, la ubicuidad de Internet y la Red global hacen realidad algo que es mucho más que una revolución tecnológica. Suponen transformaciones en la conciencia, en los hábitos perceptivos y de expresión, de sensibilidad recíproca, que apenas estamos empezando a calibrar [...] La influencia en el proceso de aprendizaje es ya trascendente.

En su consola, el colegial entra en mundos nuevos. Lo mismo hace el estudiante con su ordenador portátil y el investigador navegando en la Red. Las condiciones de intercambio colaborador, de almacenamiento de memoria, de transmisión inmediata y representación gráfica han reorganizado ya numerosos aspectos de la Wissenschaft.

La pantalla puede enseñar, examinar, demostrar, interactuar con una precisión, una claridad y una paciencia superiores a las de un instructor humano. Sus recursos se pueden difundir y obtener a voluntad. No conoce el prejuicio ni la fatiga. A su vez, el aprendiz puede preguntar, objetar, replicar, en una dialéctica cuyo valor pedagógico tal vez llegue a superar al del discurso hablado (STEINER, 2004:169).

En este complejo entramado, surge la necesidad de reflexionar sobre varios aspectos en torno a los cambios tecnológicos y se plantean nuevas propuestas pedagógicas para tratar de dar respuesta a las nuevas necesidades educativas.

\section{LA PEdagogía del Ciberespacio una teoría educativa para ENTORNOS DIGITALES}

La pedagogía del ciberespacio es un constructo conceptual que surge en el año 2011, con la idea de validar su propuesta epistemológica y constituirse como teoría de enseñanza y aprendizaje para la sociedad red ${ }^{1}$. 
Parte de la fundamentación teórica de la pedagogía del ciberespacio se sostiene en el uso de la "Teoría total de la realidad" de David Deutsch (1990), según este autor, cuando salen a la luz nuevas teorías no deben ser desplazadas las teorías antecesoras, sino que deben ser integradas al nuevo esquema conceptual.

Modelos educativos como el conductismo, cognitivismo y constructivismo - concretamente, sus teorías- han permitido orientar la explicación del objeto de estudio del campo educativo, que históricamente ha reflexionado sobre cómo se dan los procesos de enseñanza y aprendizaje; sin embargo, estos dispositivos conceptuales no han logrado analizar cómo se da el acto educativo en la sociedad red.

El aporte que pretende brindar esta teoría no sólo está en explicar cómo suceden los fenómenos educativos en una sociedad mediada por tecnologías digitales, sino que pretende complementar lo que los otros constructos conceptuales no lograron aportar por su dinámica y momento histórico.

Otro de los postulados clave para el diseño de este constructo teórico, de la pedagogía del ciberespacio, ha sido el aporte de Pierre Lévy y su categoría de análisis, denominada La inteligencia colectiva. Esta categoría defiende la idea de que las máquinas solo almacenan datos e información, y la inteligencia humana permite construir saberes sociales y aprendizajes.

El tercer aporte fundamental a las bases de esta teoría es el trabajo del psicopedagogo ruso Alexéi Leontiev, con su "Teoría de la actividad", que reconoce que los motivos educativos se obtienen, en primer lugar, a partir de la incorporación de operaciones, como actividades simples y rutinizadoras; en segundo lugar, a partir de la adquisición de acciones, como la suma de operaciones, pero que tienen un nivel de organización, y, en tercer lugar, a partir del nivel de actividades, como la suma de operaciones y acciones que apuntan al logro de resultados del aprendizaje (vilLAMAR, 2003).

El haber sustentado la base conceptual de la pedagogía del ciberespacio desde aportes de Andrés Hermann (2011) con el esquema epistémico de la teoría de la actividad permitió establecer una analogía para determinar, como base teórica central de esta pedagogía, la idea -que parte de una crítica al determinismo tecnológico- mediante la cual se establecen los siguientes aspectos: que lo que se encuentra en internet no son motivos o elementos

como los ordenadores y la red internet. Desde la Perspectiva de Manuel Castells (2004), se entiende que este momento histórico propone el paso de un modelo de economía de bienes tangibles hacia un modelo de economía de servicios o intangibles, sustentados por la producción intelectual y mercantil de la gestión del conocimiento. 
educativos, sino que, en un primer momento, se concibe como datos, como aquellas representaciones numéricas o lingüísticas fragmentadas y atomizadas que se encuentran en la realidad, para, en un segundo momento, pasar al logro de la información como la suma de datos que tienen un nivel de estructuración y pertinencia; en un siguiente y tercer momento, se presenta el nivel de conocimiento que integran los datos, la información, junto con el proceso mediación educativa y el trabajo basado en la inteligencia colectiva, que apunta a la adquisición de motivos educativos. El último momento, el cuarto, es el aprendizaje y reúne las tres condiciones antes expuestas, pero con el añadido de que estos elementos tendrán que ser transferidos o aplicados en situaciones y contextos socioeducativos reales.

Esta nueva teoría pedagógica entiende que el uso de la tecnología per se no garantiza el logro de los resultados de aprendizaje, ya que lo que se encuentra en el ciberespacio en primera instancia no es conocimiento, sino datos e información, insumos que tendrán que ser analizados e inferidos hasta convertirse en conocimiento y aprendizajes significativos.

Se ha señalado que los objetivos principales de este trabajo se orientan en determinar el impacto de la red internet y las tecnologías en los procesos educativos, así como establecer la manera en la que se dan los procesos de lectoescritura en los escenarios digitales. Pero esta reflexión estaría inacabada, sino se hubiera considerado, además, brindar un sustento epistemológico a partir de la aplicación de una teoría de enseñanza y aprendizaje que se ha relacionado con la pedagogía del ciberespacio.

Es capital entender cómo se dan las formas comunicacionales en el ciberespacio a partir de aspectos como las narrativas digitales y su aplicación, que no pasa por el uso artefactual de la tecnología, sino por la promoción de la denominada alfabetización digital desde el desarrollo del pensamiento crítico y creativo, o lo que Cassany (2012) ha referido, en términos coloquiales, como separar el grano de la paja.

Uno de los varios retos que se ha planteado la pedagogía del ciberespacio ha sido, asimismo, entender cómo operan las nuevas narrativas digitales, las cuales no se acercan a los lenguajes tradicionales, que se dan con discursos de manera lineal o secuencial, sino de forma reticular o hipertextual, como narrativas que proponen formas diferentes de interpretar y comprender los textos, textos que pueden funcionar de forma bifurcada y que pueden ser ampliados con varios recursos en línea.

Parte de la misión que tiene la pedagogía del ciberespacio es reflexionar los nuevos procesos de lectoescritura en los escenarios digitales, establecer estrategias que enfoquen la idea de que el espacio de clase ya no sólo 
se reduce al aula física o al entorno virtual de aprendizaje, sino que está en otros espacios o plataformas tecnológicas, lo que genera una experiencia de aprendizaje enriquecida, abierta, descentralizada y mediática.

Con la idea de establecer una conclusión final, vinculada al análisis de cuáles son las nuevas narrativas digitales que hacen posible las formas de leer y escribir en ambientes mediados por el uso de las tecnologías, se reflexiona en torno a la idea de que la estrategia formativa no solo deberá pasar por una reflexión lingüística, comunicacional y artefactual, sino que deberá tener una implicación sociocultural, en donde converja, como refirió Jenkins (2008), la necesidad de establecer condiciones: la articulación de los viejos medios con los nuevos medios (convergencia), promover una cultura de la participación e impulsar el desarrollo de la inteligencia colectiva, reconociendo que el conocimiento no solo viene de los expertos y de arriba, sino también desde los espacios invisibles y descentralizados, como el ciberespacio, donde nadie sabe todo, pero todos saben algo (LÉvy, 2009).

Vale recalcar que por convergencia se entiende el flujo de contenidos a través de múltiples plataformas mediáticas, la cooperación entre múltiples industrias mediáticas y el comportamiento migratorio de las audiencias mediáticas, dispuestas a ir a casi cualquier parte en busca del tipo deseado de experiencias de entretenimiento (JENKINS, 2008).

\section{PANORAMA EDUCATIVO EN EL ESCENARIO DIGITAL: EL CASO DE LA LECTURA EN RED}

El paso del uso del internet destinado para fines políticos y de inteligencia militar, hacia la vinculación de los procesos sociales en la década de los 90 del siglo XX permitió repensar las dinámicas que se estaban tejiendo en las actividades del ser humano, como la economía, la industria cultural, el entretenimiento, las comunicaciones y la educación. Con respecto a este último punto, se hace necesario repensar los procesos de enseñanza y aprendizaje que se estarían gestando para una sociedad que hace uso de tecnologías digitales y que va generando la apertura hacia la digitalización y de la construcción de una escuela 2.0 y 3.0.

Los cambios que traería el uso de la red internet y los ordenadores no sólo se circunscriben a las dinámicas educativas en la adquisición y construcción de los conocimientos, aprendizajes y saberes sociales, sino que atraviesan cambios profundos en escenarios como lo comunicacional y con esto, con la lectura y la escritura en el escenario digital. 
Para Sara Osuna (2011), el paso de la comunicación desde los flujos informacionales de las tecnologías analógicas como la televisión, la radio, la prensa escrita, hacia las tecnologías digitales representadas por la red internet y los ordenadores posibilitan el cambio de un modelo comunicacional unidireccional (uno a todos) hacia un modelo comunicacional multidireccional (todos a todos), lo que permite de alguna forma impulsar la idea de una comunicación horizontal y con esto, de una educación más descentralizada e inclusiva, sin tomar en cuenta que no se puede pensar de igual manera que todos los niños y jóvenes nacidos en generación digital pueden ser considerados nativos digitales, ya que, “contextos diferentes, antecedentes y biografías distintos, perfiles socioculturales diversos y distribución desigual de capitales condicionan los procesos de apropiación de las TIC" (BENÍtez y otros, 2012:57).

El nivel sociocultural fomenta una brecha no solo en los estudiantes, sino de las instituciones educativas., por ello es necesario tomar en cuenta ir más allá de una visión técnica, permitiendo reflexionar en torno a que "la tecnología no sea una trampa. Que los condicionamientos económicos y sociales no impidan la implantación real de estos nuevos medios en las distintas sociedades" (GARCíA, 2006:7). Hay que señalar en este punto que el contenido vinculado a los procesos institucionales "contribuye a modelar nuestras formas de vida y de ordenamiento institucional" (sNow, 1964 citado por Marino y otros, 2001:146).

\subsection{LA ACTIVIDAD DE LEER EN LA ERA DIGITAL}

A partir de los planteamientos anteriores, se puede argumentar que la actividad de leer y escribir -que no cambia, en esencia, del medio físico al virtual y que está acorde con la intencionalidad de que el sujeto construye significados, símbolos, abstracciones y un pensamiento formal- si debe tomar en cuenta que en el actual escenario tecnologizado se requiere la adquisición de competencias educativas y de procesos vinculados con la alfabetización digital. "Estas diferencias no describen solo nuevas maneras de leer y escribir, sino también modos distintos de acceder, usar, construir y concebir el conocimiento, que pueden constituir un cambio de paradigma cultural" (CASSANY, 2012, p.26).

El primer presupuesto del que se debe partir, por lo tanto, es la respuesta a qué significa leer: leer significa cerrar perceptivamente un acto cuyo inicio ha sido la estructuración textual de una información determinada. Se lee porque alguien ha preparado unos textos, de una determinada forma, para 
ser leídos. Esto implica toda una serie de decisiones previas al hecho de la lectura, decisiones formales y estructurales, de las que leer es el acto final. Leer requiere unos actos preparatorios que permitan el acople del lector con el texto. No basta con crear textos, hay que formar lectores competentes para ese tipo de textos (AGUIRRE, 2011: 25).

El segundo presupuesto insoslayable es el discernimiento de lo que implica la lectura digital. Este tipo de lectura se desarrolla en cualquier tipo de pantalla mediante el uso de un programa que permite visualizar correctamente los contenidos: textuales, iconográficos o audiovisuales. Se trata, [por lo tanto], de un tipo de lectura que se desarrolla en diferentes tipos de dispositivos con alternancias, según los textos y las prestaciones que ofrecen los mismos. Tablet, ereader o dispositivos dedicados, que emplean la tinta electrónica, smarphones y ordenadores, tanto portátiles como de mesa, son los principales espacios en los que se desarrolla la actividad lectora. La lectura digital se puede desarrollar en diferentes entornos y con diferentes posibilidades dependiendo del dispositivo, el programa o la aplicación de lectura, la red en la que se inscriba la obra, y los sistemas de DRM (Digital Rights Management o Gestión de Derechos Digitales) que fijan los usos permitidos de la misma (CORDón y otros, 2013).

Leer y escribir en el escenario digital implica interpretar, decodificar símbolos y generar códigos y significados sociales, pero, además, en la actual era digital esta actividad implica manejar códigos vinculados con herramientas tecnológicas que posibilitan dar un paso de la linealidad del texto hacia la bifurcación y construcción de discursos paralelos a la realidad.

En el texto de Daniel Cassany, En línea: leer y escribir en la red, se expone a manera de propuesta unas condiciones para determinar qué implica saber leer y escribir en los escenarios virtuales a partir de aspectos como a) la hipertextualidad: entendido como vínculos comunicantes que tienen distintos itinerarios y rompen la linealidad del discurso; b) la intertextualidad: que se relaciona con las ideas que se conectan entre sí, con los nodos y vínculos externos; c) la multimodalidad: que se basa en la conformación de varios códigos, como imágenes, sonidos, letras, etc.; y d) el plurilingüismo y la multiculturalidad: que es la condición que refiere la posibilidad de interactuar con varios interlocutores a escala global.

Lorenzo Soccavo (2011), asimismo, afirma que los lectores digitales discurren por el texto con "una lectura fragmentada, consecuencia de una lectura enriquecida: menos lineal, más extensa, de zapping, más abierta a lo multimedia; [es, también] una lectura social, consecuencia del desarrollo de las redes sociales: comentada, compartida, enriquecida por las contribuciones de los 
lectores...; [y, además,] una lectura conectada, consecuencia de una lectura más extensa y más móvil". (Soccavo, 2011, p.4).

El concepto de lectura conectada, desarrollado por Soccavo, ahonda en la posibilidad de leer sin solución de continuidad en cualquier dispositivo de lectura que tenga una conexión a Internet. Según este autor, la lectura conectada es el corolario de una mayor movilidad y de las posibilidades planteadas por el cloud computing: un modo de lectura [en directo o] in streaming desarrollado sobre el modelo que ya había sido experimentado con la música. (CORDÓN y otros, 2013).

Los rasgos de la lectura presentados anteriormente permiten evidenciar que las acciones de leer y escribir en los entornos digitales, no solo pasan por saber manejar los nuevos códigos informatizados, sino que se debe tomar en cuenta que para que los actores educativos puedan comunicarse en la red Internet, es importante considerar la disponibilidad de millones de recursos, y que los receptores y emisores podrán interactuar con una comunidad extensa de usuarios a nivel global. "Comunicar en la red es bastante más complicado porque nos relacionamos con una audiencia planetaria, con culturas y referentes muy variados". (CASSANY, 2012, p. 62).

Así mismo, el usuario que decide enfrentarse al texto digital con su fragmentariedad, movilidad, conectividad, etc., debe comprender ese texto, siempre y cuando su intención sea la comprensión. La comprensión lectora (literacy) es el paso necesario para discernir todo ese conocimiento que ofrece la red. La comprensión lectora es, además, la habilidad para entender, evaluar, utilizar e interactuar con textos escritos con el fin de participar en la sociedad, alcanzar los objetivos propios y desarrollar el conocimiento y el potencial de uno mismo (PIAAC, p.15). Por eso, es sumamente importante "asegurar la adquisición de una correcta competencia lectora, [porque] continúa constituyendo el paso previo para el dominio de los discursos existentes y de los generados a partir de los nuevos parámetros de escritura" (Morales, 2015, p.68), como los que ofrece Internet y con los que, mal que bien, los estudiantes construyen buena parte de sus trabajos de curso.

\subsection{EL HIPERTEXTO: NUEVO FORMATO TEXTUAL Y NUEVAS NARRATIVAS}

Como parte del metalenguaje que ha traído consigo el uso de la red Internet y los ordenadores en los procesos sociales, culturales y educativos, se pueden encontrar términos como: discursos multimediales, hipertextuales, hipermediales y, más recientemente, transmediáticos. Antes de iniciar con la 
definición de cada una de las categorías de análisis, este trabajo se centrará en la reflexión del término hipertexto que, desde el aporte de autores como George Landow (2006), se entiende como una suma de bloques de palabras electrónicas que se encuentran unidas en diferentes trayectos. Si bien es cierto que en esta definición se ha referido al hipertexto como característica de tipo electrónico, es importante destacar que puede haber hipertextos de tipo tradicional, como los que pueden darse en ámbitos como la literatura. Buena muestra de ello es la novela de Julio Cortázar Rayuela, relato hipertextual que propone al lector diferentes caminos o trayectos para seguir el desenlace de la historia bifurcada.

El hipertexto constituye también una forma de narrativa digital que, en el caso de la Educación, ha permitido replantear las formas comunicacionales que hacen posible la transferencia de conocimiento y la mediación del aprendizaje, ya que rompe con la linealidad del discurso que se propone en los sistemas educativos de corte tradicional.

Los actuales actores educativos deberán considerar, al momento de diseñar experiencias formativas para entornos que hacen uso de tecnologías, el uso de narrativas digitales a partir del texto, en donde gran parte del discurso o de las enseñanzas deberán reposar en enlaces, nodos, redes, como espacios que permiten un intercambio cognoscitivo de carácter abierto, ubicuo y distribuido (LANDOW, 2006).

En lo que concierne al uso del término hipertexto, en la literatura, como en el ensayo digital y en un sinfín de géneros, se utiliza como sinónimo de otros vocablos como lo multimedial e hipermedial. Con la idea de precisar los términos que son parte de las nuevas narrativas digitales, lo multimedial es la combinación de lenguajes visuales y sonoros, mientras que el hipertexto se refiere a la suma de bloques de texto que integran subtextos que pueden bifurcarse y permiten continuar los trayectos por diferentes itinerarios $o$ caminos.

Hipermedial se entiende como la suma de las dos categorías antes presentadas: la condición que integra discursos visuales, sonoros y de textos abiertos y bifurcados (BELLOCH, 2006).

Una vez que se han logrado establecer las diferencias entre algunos de los tipos de las narrativas digitales es relevante advertir que los actores educativos deben entrar en un proceso de alfabetización digital, no solo para manejar los discursos comunicacionales que permitirán garantizar el diálogo y el intercambio educativo, sino que se tendrán que fijar didácticas de enseñanza y estrategias de aprendizaje específicas, ya que estudios en el campo de la neurociencia refieren que el uso de lenguajes visuales y sonoros permite a los 
educandos estimular las estructuras cognitivas y activar dopaminas, lo que se traduce en potenciar los procesos de aprendizaje desde condiciones fisiológicas y motivacionales en la mente humana. (WATSON, 2011).

El uso de los diferentes códigos o medios en los que se presenta la información viene determinado por la utilidad y funcionalidad de los mismos dentro del programa. Y la inclusión de diferentes medios de comunicación -auditivo, visual- facilita el aprendizaje y se adapta en mayor medida a los sujetos, sus características y capacidades - pueden potenciar: memoria visual, comprensión visual, memoria auditiva, comprensión oral-. (BELLOCH, 2006).

La importancia de establecer la definición de lo que representa cada una de las narrativas digitales aporta no solo en el sentido de determinar las características de lo que implica leer y escribir en los nuevos ecosistemas, como son los entornos digitales que se representan a partir de algún tipo pantalla, sino que permite mostrar el uso y la funcionalidad en términos de enseñanza y de aprendizaje. Belloch (2006) afirma que un adecuado proceso de decodificación y lectura en los entornos digitales se da a partir de diferentes tipos de navegación:

a) Sistema lineal: la lectura se da en un solo recorrido o trayecto secuencial.

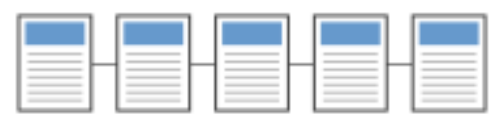

FIGURA 1: LECTURA SECUENCIAL TOMADA DE BELLOCH (2006)

b) Reticular: este tipo de navegación se caracteriza por manejar un tipo de texto abierto y bifurcado, donde el lector tiene la posibilidad de navegar a partir de diferentes itinerarios, los cuales se ajustan a sus necesidades e intereses socioeducativos.

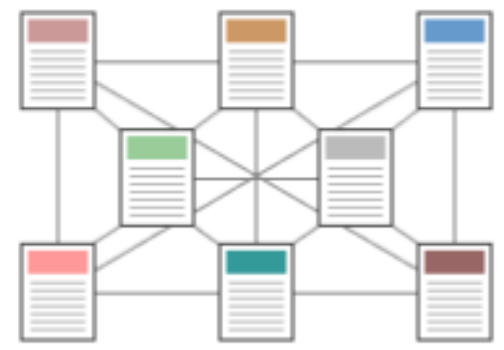

FIGURA 2: LECTURA RETICULAR TOMADA DE BELLOCH (2006) 
c) Jerarquizado: entre las ventajas que presenta este tipo de navegación está el nivel de organización de los bloques de datos e información, aunque no permite al lector o usuario escoger los trayectos narrativos. En el caso del diseño para propuestas en entornos educativos mediados por tecnologías, se sugiere elaborar objetos de aprendizaie de tipo reticular.

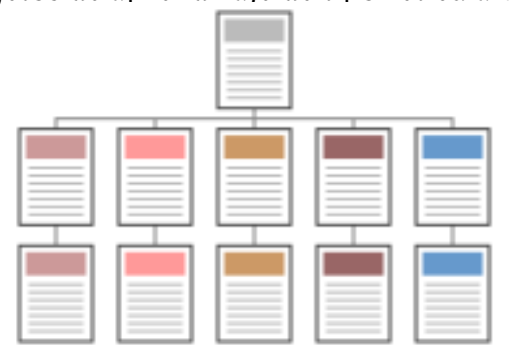

FIGURA 3: LECTURA JERARQUIZADA TOMADA DE BELLOCH (2006)

Los cambios educativos en el contexto de uso de las tecnologías tendrán que incorporar no solo el manejo artefactual de las herramientas, sino también generar procesos de alfabetización digital en la decodificación y la interpretación de las nuevas narrativas digitales.

Sobre la base de las ideas expuestas anteriormente, en los sistemas de navegación se puede inferir que la educación tradicional o que hace uso de las tecnologías analógicas favorece por sus formatos de texto las lecturas lineales o secuenciales, pero en el caso de la educación que hace uso de tecnología y posee una gran cantidad de recursos, se tendrá que enseñar a los nativos digitales la interpretación de lecturas reticulares e hipertextuales y se deberá capacitar a los docentes y educandos en habilidades para el procesamiento de datos e información hacia la construcción del conocimiento del aprendizaje.
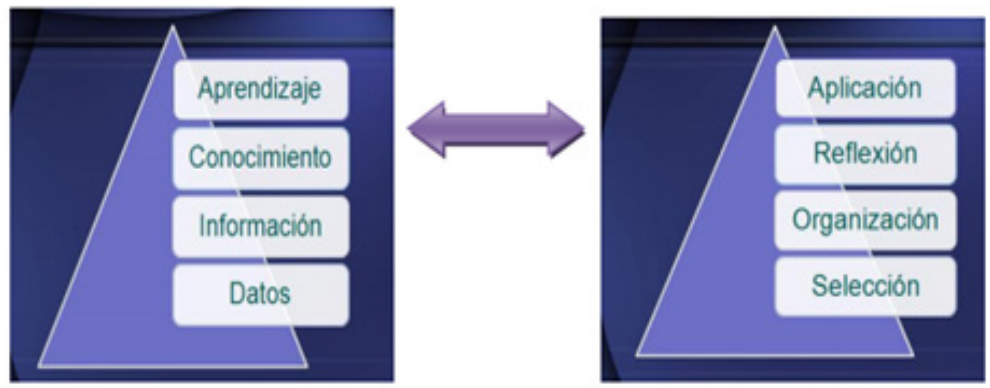

FIGURA 4. ELABORACIÓN PROPIA: MODELO DE PROCESAMIENTO ESQUEMA PEDAGOGÍA DEL CIBERESPACIO 


\section{CONCLUSIONES}

En la era digital, la sociedad debe revisar con profundidad qué aspectos del conocimiento se están transformando con la entrada masiva de lo digital en la cotidianidad de los usuarios, como mencionan Apolo y otros (2014). Es relevante, entonces, sobrepasar enfoques instrumentalistas ubicados en el determinismo tecnológico hacia procesos de construcción colectiva de procesos educativos.

Es necesario, además, establecer líneas de investigación hacia la construcción social de un sentido de legitimidad en procesos de enseñanza aprendizaje mediados por tecnologías, para comprender los procesos socioculturales que interactúan en los mismos. Desde esta perspectiva, cabe poner en diálogo la triada comunicación, cultura y desarrollo, tal como subraya Ulloa (2007), y no solo la diada comunicación y tecnología, como prototípicamente se analiza y reduce el debate en la enseñanza de lo digital.

El paradigma de la web 2.0 ha permitido un rol activo de los usuarios de la red internet, ya que estos, con el uso de recursos como las redes sociales y los entornos digitales, han pasado de ser consumidores a productores de contenidos, ideas y significados. En el momento actual se está configurando un modelo web 3.0, en donde el rol de Internet se caracteriza por ser semántica, ya que los usuarios pueden cocrear contenidos de manera colectiva en el ciberespacio, inaugurando así lo que se denomina una web aumentada o enriquecida.

La educación en un contexto como el actual debe integrar conocimiento y técnica para evitar, así, que lo instrumental no predomine ante lo cognoscitivo, el pensamiento crítico y la reflexión. La educación debe favorecer la sinergia positiva, resultado de una pedagogía que potencie lo analítico y lo tecnológico.

La pedagogía del ciberespacio tiene en cuenta este nuevo escenario en el sistema educativo y fomenta una reflexión a partir de la integración de presupuestos teóricos tradicionales y nuevas aportaciones teóricas derivadas de la sociedad red.

La lectura tiene un papel preponderante en este contexto porque la habilidad o competencia para poder procesar con criterio la enorme cantidad de datos de la red tendrá consecuencias en el aprendizaje de los discentes y, en última instancia, en el desarrollo de la sociedad red. Por esta razón, el educando y, en general, el usuario debe comprender bien los nuevos formatos textuales digitales.

La pedagogía del ciberespacio podría constituir un dispositivo conceptual 
que brinde explicaciones en torno a cómo se deben generar los procesos de enseñanza y aprendizaje en la sociedad red; además, este constructo posibilitaría orientar la práctica de leer y escribir en entornos digitales, ya que sostiene la importancia de identificar cómo se procesan los datos, información, conocimientos y aprendizajes.

Los procesos de lectura y escritura en la red han dado un paso de la narración lineal o secuencial hacia una narración de tipo reticular, hipertextual o bifurcada, donde los sujetos pueden decidir el itinerario o trayectoria del relato, lo que permite, además, una lectura ampliada, enriquecida y que responde a los intereses y necesidades del lector.

Internet ha planteado nuevas formas de narrativas digitales en las que las lógicas de interpretación y codificación de los datos e información se dan a partir de recursos multimediales, hipertextuales, hipermediales y transmediáticos, que refieren la idea de que la lectura y escritura debe integran lenguajes visuales, sonoros, sensoriales, que pueden ser obtenidos en diferentes canales mediáticos o plataformas tecnológicas.

\section{BIBLIOGRAFía}

AGUIRRE, Joaquín (2011). "La transformación de la sociedad lectora con el impacto tecnológico: el futuro lector" en MONTESA, Salvador (Coord.)., Literatura e internet, nuevos textos, nuevos lectores, Málago: Actas del XX Congreso de Literatura Española Contemporánea Universidad de Málaga.

APOLO, Diego; ALTAMIRANO, Ma. Victoria; VÁSCONEZ, Verónica; CEVALLOS, Ma. Isabel (2015). "Usuarios, clientes y consumidores digitales: consideraciones para su abordaje desde el marketing y la comunicación corporativa". Revista Redmarka: revista académica de marketing aplicado, número 14, Unidad de Investigación en Marketing Aplicado (UIMA)-Universidad de A Coruña - Facultad de Ciencias de la Comunicación de la Universidad de A Coruña pp. 3-19.

BAUMAN, Zygmunt (2007). Los retos de la educación en la modernidad líquida, Barcelona: Gedisa.

BELLOCH, Consuelo (2006). Aplicaciones Multimedia Interactivas: Clasificación, Valencia: Unidad de Tecnología Educativa, Universidad de Valencia. Dirección: http://www.uv.es/bellochc/pdf/pwtic3.pdf (Última consulta: 07 de diciembre de 2015).

BENÍTEZ LARGHI, Sebastián, AGUERRE, Carolina; CALAMARI, Marina; FONTECOBA, Ariel; MOGUILLANSKY, Marina; PONCE DE LEÓN, 
Jimena (2011). "De brechas, pobrezas y apropiaciones. Juventud, sectores populares y TIC en la Argentina". Revista Versión, Estudios de Comunicación y Políticas, volumen 22, número 32, Universidad Autónoma Metropolitana de México, Xochimilco, pp. 1-20.

BONAL, Xavier. (1998). Sociología de la educación: una aproximación crítica a las corrientes contemporáneas, Barcelona: Paidós Ibérica.

CASSANY, Daniel (2012). En línea: leer y escribir en la red, Barcelona: Anagrama.

CASTELLS, Manuel (2004). La era de la información: economía, sociedad y cultura. Economía, sociedad y cultura, México: Siglo XXI.

CORDÓN, José (coord.) (2013). "Diccionario Digital de Nuevas Formas de Lectura y Escritura”, Salamanca: Ediciones Universidad de Salamanca y Red Internacional de Universidades Lectoras. Dirección: http://dinle.eusal.es/ index.php (Última consulta: 11 de agosto de 2015).

DEUTSCH, David (1999). La estructura de la realidad, Barcelona: Anagrama.

GARCÍA, Francisco (2006). "Contenidos educativos digitales: Construyendo la Sociedad del Conocimiento". Red digital: Revista de Tecnologías de la Información y Comunicación Educativas, volumen 6, número 1, Ministerio de Educación y Ciencia de España, Madrid, pp. 1 - 29

HERMANN, Andrés (2011). "Pedagogía del ciberespacio: hacia la construcción de un conocimiento colectivo en la sociedad red". Revista Sophia, número 11, Editorial Universitaria Abya-Yala, Quito: pp. 83-103.

JENKINS, Henry (2008). La cultura de convergencia de medios de comunicación, Barcelona: Paidós.

JENKINS, Henry (11 de febrero de 2009). "If it doesn't spread, it's dead (part one): media viruses and memes". Confessions of an ACA-Fan-The Official Weblog of Henry Jenkins. (Traducción colaborativa Cátedra de Procesamiento de Datos, 2010) Dirección: http://proyectoredisenar.pbworks.com/ TRADUCCION-If-It-Doesn\%27t-Spread\%2C-It\%27s-Dead-\%28PartOne\%29\%3A-Media-Viruses-and-Memes (Última consulta: 16 de abril de 2015).

KRAWCZYK, Nora (2002). "La reforma educativa en América Latina desde la perspectiva de los organismos multilaterales". Revista Mexicana de Investigación Educativa, volumen 7, número 16, Consejo Mexicano de Investigación Educativa A.C, pp. 627-663.

LANDOW, George (2009). Hipertexto 3.o. Teoría crítica y nuevos medios en la era de la globalización, Barcelona: Paidós Ibérica.

LÉVY, Pierre (2009). Inteligencia colectiva por una antropología del ciberespacio. Washington, Dc.: Organización Panamericana de la Salud. 
MARINO, Eduardo (2001). Ciencia, tecnología y sociedad: una aproximación conceptual, Madrid: Cuadernos de Iberoamérica, Organización de Estados Iberoamericanos para la Educación, la Ciencia y la Cultura.

MORALES, Ma. Isabel (2015). "El reto de la formación transversal en la universidad: lectura, escritura y nuevas tecnologías” en Ramírez, Elsa (Coord.)., Tendencias de la lectura en la Universidad, México DF.: UNAM, Instituto de Investigaciones Bibliotecológicas y de la Información.

OSUNA, Sara (2011). "Aprender en la web 2.o. Aprendizaje colaborativo en comunidades virtuales” en: Revista La Educ@ción, Portal Educativo de las Américas - Departamento de Desarrollo Humano, Educación y Cultura, pp. 1-19. Dirección:

http://www.educoas.org/portal/La_Educacion_Digital/laeducacion_145/articles/ART_osuna_ES.pdf (Última consulta: 20 de febrero de 2015).

RAMA, Claudio (2004). "El nuevo escenario de la educación superior en América Latina y la educación virtual" en Rama, Claudio (coord.) La educación superior virtual en América Latina y el Caribe, México, Df.: Serie Memorias, Colección Biblioteca de la educación superior, Asociación Nacional de Universidades e Instituciones de Educación Superior.

SNOW, Charles (1977): Las dos culturas y un segundo enfoque. Madrid: Alianza Editorial.

SOCCAVO, Lorenzo (2011), "Comprendre la 4e révolution du libre". Enjeux pour l'interprofession,Congrès i-expo 2011, organisé par le GFII,Session Economie de la connaissance etlivres numériques: quels modèles économiquespour quels usages ? Dirección: http://www.i-expo.net/uploads/2011/actes/ $\mathrm{P}_{5}$ SOCCAVO_Lorenzo_contrib_iexpo2011.pdf (Última consulta: 13 de enero de 2015).

STEINER, George (2004). Lecciones de los maestros, Madrid: Ediciones Siruela. ULLOA, Cesar (2007). Comunicación, cultura y desarrollo, Quito: Quipus.

VILLAMAR, Feliciano. (2003). Psicología evolutiva y psicología de la educación. Barcelona: Universidad de Barcelona. Dirección: http://www.ub.edu/dppsed/fvillar/principal/pdf/proyecto/cap_01_evolutiva_y_educacion.pdf (Última consulta: 08 de marzo de 2015).

WATSON, Richard (2011). Mentes del futuro. ¿Está cambiando la era digital nuestras mentes?, Barcelona: Editorial Viceversa. 